\title{
Light Fidelity Technology
}

\author{
Amrit Jyot Singh, Abhilash Veeraiahgari \\ UG Student Department of E.C.E, $K$ L University \\ UG Student Department of E.C.E, K L University
}

\begin{abstract}
Light-Fidelity explains how visible light communication (VLC) technology is applied to high speed wireless communication. Light-Fidelity technology was first demonstrated by Harald Hass, a German Physicist. DLIGHT, one of his invention which can produce data rates faster than 10 megabits per second, which is speedier than your average broadband connection. Light-Fidelity is used to increase wireless networks in places such as home, office, business environment. Using Light-Fidelity technology, in future data for laptops, smart phones, and tablets can be transmitted through the light in a room.
\end{abstract}

Key words: Light-Fidelity, VLC, DLIGHT

\section{Introduction}

An alternative of RF wireless technology is Optical wireless technology. As this technology offers a huge bandwidth which is unlicensed so can be used for many applications such as streaming video and music, access of Internet, etc.

The data can be transmitted through LED Light which is presently observed in Electronic Devices like Ham Radios, Television Remotes, etc.

As the number of devices connected to a particular wireless network increases the speed of the network decreases. So, In order to overcome this problem Data transmission through LED is done. Generally data is transmitted through EM waves or else through Data Illumination.

In future data can be transmitted through the light in a room for laptops, smart phones, and tablets.

\section{About Li-Fi Technology}

The idea of Li-Fi was first given by Harald Haas from University of Edinburgh, UK, in his TED Global talks on Visible Light Communications.

$\mathrm{Li}-\mathrm{Fi}$ is transmission of data through illumination by taking the fiber out of fiber optics, sending data through an LED light bulb that varies in intensity faster than the human eye can follow. He explained, "Very simple, if the LED is on, you transmit a digit 1, if it is off you transmit a 0. The LEDs can be switched on and off very quickly, which gives nice opportunities for transmitting data." So the requirement is LEDs and a controller that code data into those LEDs.

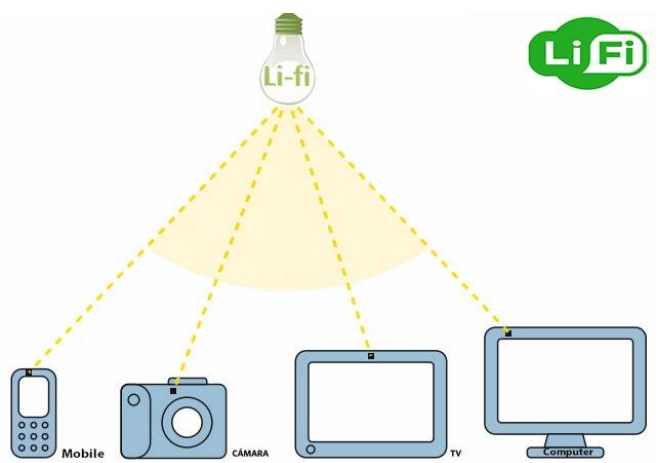

Figure 1: Li-Fi Technology Usage

Depending on the data we want to encode or transmit we have to vary the rate at which the LED flickers.

Enhancements to be made in this methods are like using an array of LEDs for parallel data transmission or using mixtures of red, green and blue LEDs to change the light's frequency with each frequency encoding a different data channel.

By the above advancement we can achieve a theoretical value of speed upto 10GbPS, i.e. we can download 1 GB file in just 30 seconds irrespective of the file format.

It can be used in the place such as hospitals, traffic signals, modern medical instruments. Li-Fi can also be used underwater where Wi-Fi cannot be used. 


\section{Working of Light Fidelity:}

Light Fidelity ( $\mathrm{Li}-\mathrm{Fi})$ is a fast and reliable alternate for Wi-Fi. The Working Principle of Li-Fi is Visible Light Communication.

Visible Light Communication is a medium which uses spectrum of light ranges from $400 \mathrm{THz}$ to $800 \mathrm{THz}$ as a light carrier for data illumination and transmission.

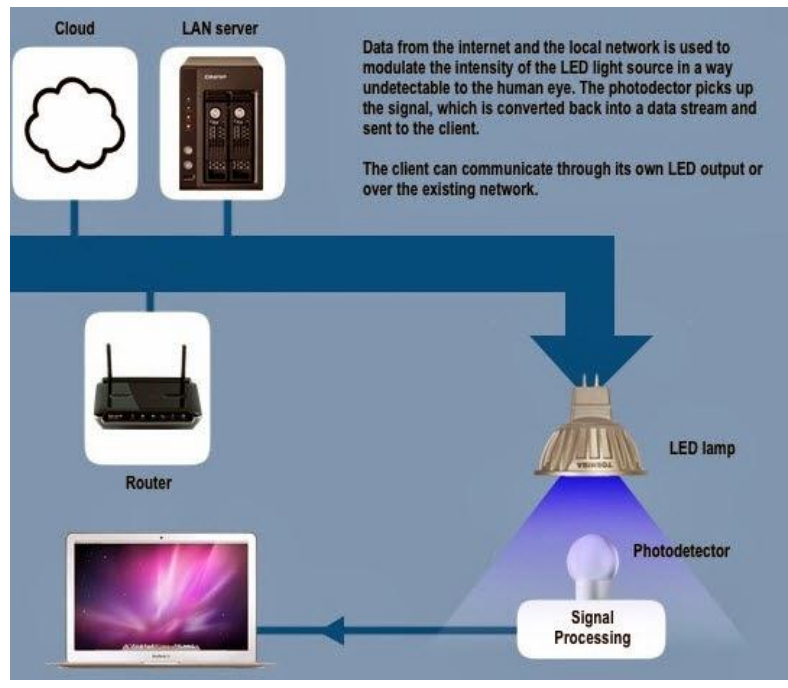

Figure 2: Working of Light Fidility

Based on the number of LEDs used, more data processing is done. This is achieved by flickering of LEDs that cannot be detected by a human eye. The flickering of LEDs create binary digits code, i.e., when LED is in ON state it transmits a binary digit One (1) and if it is in OFF state binary digit Zero (0) is transmitted.

Using proper multiplexing techniques data rate of approximately $100 \mathrm{Gbps}$ can be achieved using high speed LEDs.

VLC data rate can be increased by parallel data transmission using LED arrays where each LED transmits a different data stream. There are reasons to prefer LED as the light source in VLC while a lot of other illumination devices like fluorescent lamp, incandescent bulb etc. are available. [1](5)

$\mathrm{Li}-\mathrm{Fi}$ can be used to increase wireless networks in places such as home, office, business environment. The restriction Li-Fi technology faces is line of sight. So, it is not possible to substitute Wi-Fi.

The infrastructure of Li-Fi technology is nothing but an array of LEDs as a transmitter and a photo detector as a receiver.

\section{Comparison of Li-Fi and Wi-Fi:}

$\mathrm{Li}-\mathrm{Fi}$ is a term of one used to describe visible light communication technology applied to high speed wireless communication. It acquired this name due to the similarity to WI-FI, only using light instead of radio. WI-FI is great for general wireless coverage within buildings and LI-FI is ideal for high density wireless data coverage in confined area and for relieving radio interference issues, so the two technologies can be considered complimentary.

Table 1: Comparison of Current and Future Wireless Technologies

\begin{tabular}{|l|l|l|}
\hline Technology & Speed & Data Density \\
\hline Wireless(Current) & & \\
\hline $\begin{array}{l}\text { WI-FI } \\
\text { IEEE802.11n }\end{array}$ & $150 \mathrm{Mbps}$ & $*$ \\
\hline Bluetooth & $3 \mathrm{Mbps}$ & $*$ \\
\hline IrDA & $4 \mathrm{Mbps}$ & $* * *$ \\
\hline Wireless(Future) & & \\
\hline WiGig & $2 \mathrm{Gbps}$ & $* *$ \\
\hline Giga-IR & $1 \mathrm{Gbps}$ & $* * *$ \\
\hline LI-FI & $>1 \mathrm{Gbps}$ & $* * * *$ \\
\hline
\end{tabular}

The table also contains the current wireless technologies that can be used for transferring data between devices today i.e. Wi-Fi, Bluetooth and IrDA. Only Wi-Fi currently offers very high data rates. 
The IEEE $802.11 \mathrm{n}$ in most implementations provides up to $150 \mathrm{Mbit} / \mathrm{s}$ (in theory the standard can go to $600 \mathrm{Mbit} / \mathrm{s}$ ) although in practice you receive considerably less than this. Note that one out of three of these is an optical technology [2].

\section{Illumination of Light Fidelity Source:}

The LI-FI source has very high lumen intensity. In other words, a single source, only a few millimeters in size can produce 2300 lumens of brilliant white light. At this level of output, you will only need to use one light source per street light in most cases. This makes the mechanical and optical implementation of light much simpler and less expensive [3].

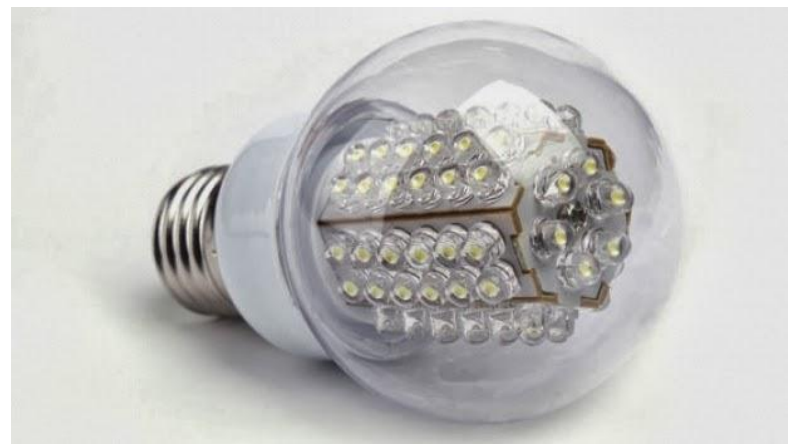

Figure 3: Li-Fi Source (LED)

\subsection{Smart Lighting:}

\section{Applications of Light Fidelity:}

Any private or public lighting including street lamps can be used to provide Li-Fi hotspots and the same communications and sensor infrastructure can be used to monitor and control lighting and data.

\subsection{Hazardous Environments:}

$\mathrm{Li}$-Fi provides a safe alternative to electromagnetic interference from radio frequency communications in environments such as mines and petrochemical plants.

\subsection{Hospital \& Healthcare:}

Li-Fi emits no electromagnetic interference and so does not interfere with medical instruments, nor is it interfered with by MRI scanners.

\subsection{Mobile Connectivity:}

Laptops, smart phones, tablets and other mobile devices can interconnect directly using Li-Fi. Short range links give very high data rates and also provides security.

\subsection{Aviation:}

Li-Fi can be used to reduce weight and cabling and add flexibility to seating layouts in aircraft passenger cabins where LED lights are already deployed. In-flight entertainment (IFE) systems can also be supported and integrated with passengers' own mobile devices.

\subsection{Toys:}

Many toys incorporate LED lights and these can be used to enable extremely low-cost communication between interactive toys.

\subsection{Underwater Communications:}

Due to strong signal absorption in water, RF use is impractical. Acoustic waves have extremely low bandwidth and disturb marine life. Li-Fi provides a solution for short-range communications.

\subsection{RF Spectrum Relief:}

Excess capacity demands of cellular networks can be off-loaded to Li-Fi networks where available. This is especially effective on the downlink where bottlenecks tend to occur 


\subsection{RF Avoidance:}

Some people claim they are hypersensitive to radio frequencies and are looking for an alternative. Li-Fi is a good solution to this problem.

\subsection{Vehicles \& Transportation:}

LED headlights and tail-lights are being introduced. Street lamps, signage and traffic signals are also moving to LED. This can be used for vehicle-to-vehicle and vehicle-to-roadside communications. This can be applied for road safety and traffic management.

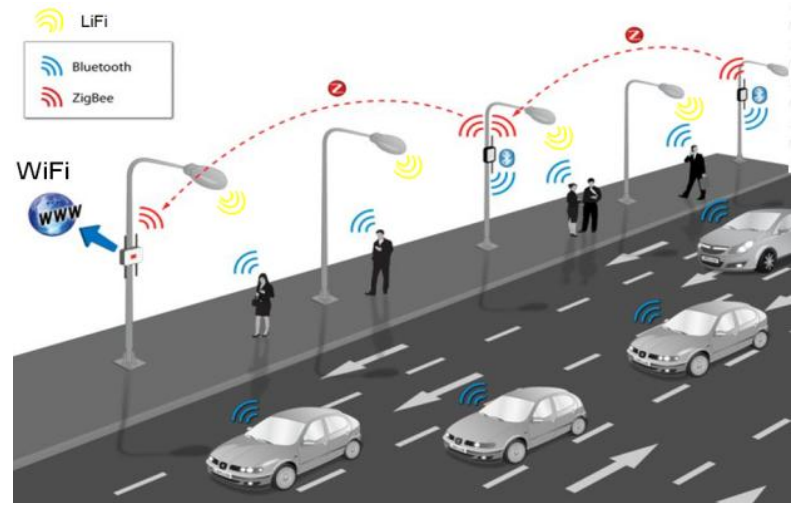

Figure 4: Li-Fi Environment.

\subsection{Location Based Services (LBS):}

Highly accurate location-specific information services such as advertising and navigation that enables the recipient to receive appropriate, pertinent information in a timely manner and location.

\section{Conclusion:}

There are many possibilities which can be explored further, in manufacturing processes to develop every LED bulb to act as a Wi-Fi Hotspot to transmit data which will lead to a Eco-friendly environment because it does not involve radio waves, as radio waves are harmful. Light Fidelity (Li-Fi) technology is wireless communication for data streaming irrespective of its formats. This type of invention can be encouraged to develop an Eco-Friendly technology.

[1] Www.lificonsortium.org

\section{References:}

[3] the-gadgeteer.com/2011/08/29/Li-Fi-internet-at-the speed-of-light/

[4] http://en.wikipedia.org/wiki/Li-Fi

[5] www.macmillandictionary.com/buzzword/entries/ Li-Fi.htm

[6] Wireless data from every light bulb Harald Haas, TED Global, Edinburgh, July 2011.

[7] Will Li-Fi be the new Wi-Fi?, New Scientist, byJamie Condliffe, dated 28 July 2011

[8] "Visible-light communication: Tripping the light fantastic: A fast and cheap optical version of Wi-Fi is coming", Economist, dated 28 Jan 2012.

[9] http://www.digplanet.com/wiki/Li-Fi. 